\title{
Perspectives of Using Ultrasonic surface hardening in Machine Part Manufacturing
}

\author{
Yulia Semenova, ${ }^{*}, 1$ and Semen Mazhuga ${ }^{1}$ \\ ${ }^{1}$ Novosibirsk State Technical University, Faculty of Mechanical Engineering and Technologies, \\ 630073 Prospekt K. Marks, 20, Novosibirsk, Russia
}

\begin{abstract}
The paper under consideration presents the review of the research results of different scientific schools on applying ultrasonic vibration energy. The effect of ultrasonic vibration energy on changes in the structure of surface layer material, surface topography and other characteristics are presented. A wide range of possibilities of using ultrasonic surface hardening in preparing surfaces before the chemicalthermal processing, for coating and as a finishing processing is presented. The possibilities of applying the method in the combination with other methods of affecting the material are considered. The possibilities of reducing the price of the machine part manufacturing because of implementing the operation of ultrasonic surface hardening in the technological process are shown.
\end{abstract}

\section{Introduction}

Ultrasonic surface hardening is used in industry since the 1950s. Both Russian and foreign researchers studied the problems of investigating and implementing the ultrasonic surface hardening in the production. The scientific papers show the possibilities of applying the method and the fundamental principles for determining the quality parameters of the surface layer through varying the processing modes for steels, cast iron and nonferrous metals. The essence of the ultrasonic surface hardening lies in the intense transfer of strike energy of the deforming tool to the surface layer material. The strikes are produced by mechanical vibrations with ultrasonic frequency. Because of little time of the tool contact with the processed metal the load on the machine part does not lead to changing its shape but acts only within the surface layer. Overlapping separate imprints controlled by changing the processing kinematic parameters allows creating a regular micro-relief. The mechanisms of micro-relief forming by mathematical modelling are studied in Papers $[1,2]$ in detail, which allowed not only explaining the essence of the processes but also predicting the processing results based on the designated technological modes. The application of the mathematical functions allows refusing the empirical choice of the processing modes and turning to determining the technological modes proceeding from the task of providing the

${ }^{*}$ Corresponding author: yu.semenova@corp.nstu.ru 
most favorable micro-relief differing in the regular geometry from the viewpoint of the operational characteristics.

Besides, the processing by ultrasonic surface hardening allows creating residual compressive stresses, which increase the fatigue durability of parts [3]. It testifies the necessity of using ultrasonic surface hardening as the finishing processing. However, it must be noted that there are difficulties in using ultrasonic surface hardening for processing thin-walled parts as well as parts with a different wall thickness $(3 \mathrm{~mm}-5 \mathrm{~mm})$. The residual compressive stresses which appear during the processing of parts with a different wall thickness can lead to the part deformation as a whole [3].

The papers devoted to investigating the effect of ultrasonic surface hardening on the wear resistance of parts processed by the combined methods are also known. The wear resistance of the samples processed by ultrasonic impact electro-spark melting treatment can be significantly improved without changing the chemical composition of the material [4]. Paper [5] presents the results of experimental investigations on improving the tribological pair "wheel - rail" by ultrasonic surface hardening, which prove its efficiency in comparison with heat-treatment. The authors explain the obtained effect by the fact that the surface hardening with a simultaneous creation of compressive residual stress. Besides, the hardened surface is less subject to the fretting-wear than the surfaces after grinding.

Nowadays one can observe the shift of research and practical interests to the possibilities of providing better operational characteristics of the surface layer by changing the metal structure in the surface layer, which considerably expands the spheres of using the method. Due to additional possibilities for modifying the surface layer, it is possible to obtain the increase in adhesion and diffusion of coatings on the surfaces processed by the ultrasonic surface hardening $[6,7]$.

Thus, the perspectives of using the ultrasonic surface hardening are rather extensive. However, to use the method of modifying the surface layer in the best way, one should understand more the nature of phenomena taking place in the material during the processing. The review under consideration is aimed at considering the mechanisms of affecting the ultrasonic surface hardening the structure and mechanical properties of the surface layer material as well as the formation of the surface topography. All the changes in the material combined determine a number of performance and technological characteristics, which will allow achieving new results in processing different materials.

\section{Changing the structure of the surface layer metal}

One of the results of the ultrasonic surface hardening effect is the grain fining of the material structure of the surface layer. The essence of the process lies in the grain fining in the poly-crystal metal or mono-crystal into zones oriented in different directions during the plastic deformation. In this case, disoriented zones have the so-called mesoscopic dimensions (values of $10^{-9} \mathrm{~m}$ ). The degree of affecting the preliminary ultrasonic surface hardening the changes in properties and structure of steel, further subject to the thermal processing, is studied in Paper [8]. In studying the influence of the ultrasonic processing on the structure and properties of heat resistant steel "ЭК-181" after the thermal treatment it is found that the dimension of the structure fragments after processing is 140 nanometers, which can be considered as a structure close to nanocrystal. According to the authors, the 
release of chrome carbide from the solid solution during the further thermal treatment decreases due to forming nanocrystal structures during the ultrasonic surface hardening. It leads to appearing a considerable residual compressive stress. In this case, the increase in the yield stress because of decreasing the recrystallization during the further ageing due to the released nanocrystals of vanadium carbide on the grain boundaries is observed. The presence of the considerable inner stresses mentioned before suppresses the pore formation and the formation of micro-cracks.

It is noted in Papers $[8,9]$ that the nano-structuring increases the static and cyclic durability and leads to increasing the strength characteristics (the yield stress, ultimate resistance and durability limit), the corrosion resistance and other properties.

Thus, the application of the ultrasonic surface hardening allows obtaining the improved mechanical properties by nano-structuring. The fact that the nanocrystal structure allows maintaining the improved properties of the part processed by the ultrasonic surface hardening even in increasing the temperatures or during the thermal processing is also significant. It considerably increases the perspectives of applying the method in industry.

\section{Creating the Favorable Micro-Geometry and Improving the Operational Characteristics}

The possibility of providing a high quality of the surface, which is characterized, first of all, by the regular micro-relief with a low roughness, is used in preparing the blank parts before the coating. Papers $[7,10]$ consider the application of the ultrasonic surface hardening as an operation, which provides the micro-relief necessary for forming the coatings with the required structure and properties. It is shown that the amplitude and spacing of microroughness affect the cooling speed and the temperature gradient of the plasma coating. In this case, it is possible to obtain the highest temperature gradient and cooling speed at the maximum amplitude and spacing of micro-roughness. Increasing the cooling speed in certain limits according to Paper [11] contributes to forming submicrocrystal phases in the metal. However, the excessive rise in the cooling speed leads to appearing the residual tension stress and increases the coating tendency to separation. Controlling the microgeometry parameters during the ultrasonic surface hardening should be considered as the possibility of creating coatings with the favorable structure and properties. Papers $[6,12]$ show the positive effect of morphology of an absolutely new regular micro-relief on spreading liquid sprayed particles and forming the drops spreading and solidifying on the surface. A specific micro-relief with a fine-grained material structure contributes to providing a reliable adhesion bond.

The investigations on obtaining a specific micro-geometry which is the most rational from the viewpoint of the further performance are presented in Papers [1, 2]. The mathematical model presented in these papers determines the morphology of a new microrelief by calculating the parameters of separate indenter imprints and setting the parameters of their overlapping during the processing (Figure 1). 


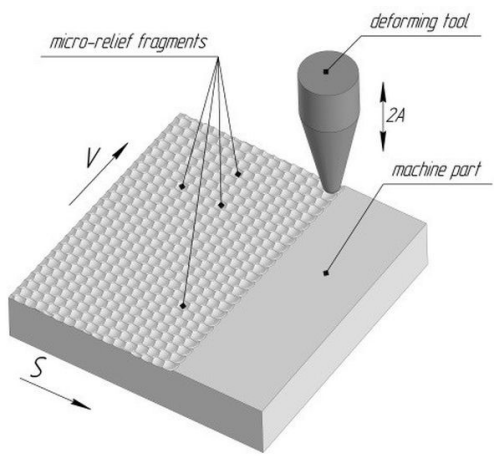

Fig. 1. Scheme of processing by the ultrasonic surface hardening.

Besides, the papers consider the possible variants of forming a new micro-relief by changing the mode parameters of the ultrasonic surface hardening (Figure 2, a-d). Using this model, it is possible to determine the rational processing modes and control the final quality of the part, which will influence its operational properties positively.

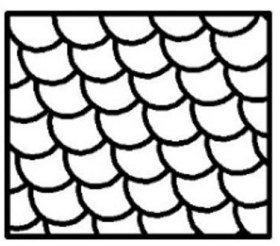

a

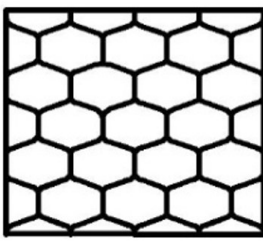

b

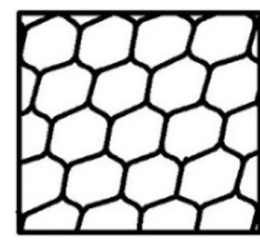

C

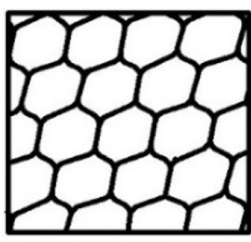

d

Fig. 2. Different shapes of the micro-relief fragments after the ultrasonic surface hardening: a micro-relief with square fragments; $\mathrm{b}$ - micro-relief with fragments of equilateral hexagon; $\mathrm{c}, \mathrm{d}-$ micro-relief with fragments of non-equilateral hexagon.

Increasing the quality of the surfaces restored by built-up welding is also considered as a perspective possibility of using the ultrasonic surface hardening. Paper [13] considers the processing of the surface restored. A low quality of and micro-geometry and the presence of pores are typical of the weld layer. It is determined that it is possible both to reduce the roughness value and increase the micro-hardness and to obtain the effect of small pore collapse and the stretching with decreasing the depth of large pores by using the surface deformation. In this case, the residual traces of small pores are not observed in investigating with a hundredfold magnification.

It follows that the ultrasonic surface hardening contributes to increasing the part durability without using additional means, providing the most favorable micro-geometry. The disadvantage of the ultrasonic surface hardening lies in the complexity of the individual selection of the processing modes for a particular material. With the appearance of the mathematical methods of processing it will stop being crucial from the viewpoint of a wide application of the ultrasonic surface hardening.

\section{Application of the ultrasonic surface hardening as a preparatory operation before the thermo-chemical processing}

In addition to controlling the micro-geometry, the ultrasonic surface hardening possesses the property of increasing the dislocation number in the processed metal. Similar 
mechanisms are interesting not only from the viewpoint of obtaining the compression stress and hardening but also from the standpoint of activating the surface layer. Papers $[6,8,14]$ study the possibilities of activating the surface layer of steels and nonferrous metals to ease the ion transfer during the thermo-chemical processing.

The mechanism of intensifying the ion transfer lies in increasing the dislocation mobility as a result of the ultrasonic surface hardening. The ultrasonic vibrations contributes to displacing the edge dislocation from one plane to another parallel to it. It is accomplished by the diffusion of interstitial atoms and vacancies to the dislocation line. This process contributes to the excessive formation of the "deformation" vacancies capable to be maintained for a long time, participating in the diffusion processes.

The ion implantation of surfaces after the ultrasonic surface hardening also leads to forming the layer of the inner nitriding, the thickness and the phase composition of which depend on the implantation temperature. The inner nitriding with decreasing the formation of the nitride continuous layer on the surface contributes to forming the particles of the nitrides of alloying elements distributed in the matrix. Their distinguishing feature is the thermodynamic stability, which contributes to stabilizing the nitride-hardened metal at higher temperatures, thus providing the effective strengthening of the material. The effect described is connected with increasing the intensity of intra-crystalline diffusion, growing the number of crystallization nucleus of nitride particles and increasing the density of the linear defects in the surface layer $[6,7]$.

The preliminary ultrasonic surface hardening is used for increasing the quality of the thermos-hardening in Paper [15]. The aim of conducting the processing is the activation of the surface layer. The preliminary ultrasonic processing contributes to increasing the plasticity of submicrocrystalline titanium with preserving high strength properties of the material.

Thus, it is possible to improve considerably the results of the further thermochemical processing by ultrasonic surface hardening. In the perspective, the increase in the diffusion capacity because of the excessive number of vacancies can be used for saturating presurface layers of the part during the ultrasonic surface hardening. It can be done by providing sufficient energy of a single indenter strike and the supply of the saturating material in the deformation zone.

\section{Combined Processing with Using Ultrasonic surface hardening}

The ultrasonic surface hardening is often used to lower the production costs of the part by providing the improved operational characteristics of typical construction materials. The example of such an application is described in Paper [16]. The ultrasonic surface hardening after conducting the electro-spark carbonizing considerably increases the operational characteristics of the part, improving the tensile strength during the sample stretching of steel $40 \mathrm{Cr}$ by 1.22 times in comparison with the samples without the ultrasonic surface hardening. Besides, the samples wear resistance increased by 7.8 times for steel $40 \mathrm{Cr}$.

The method described above found its application in manufacturing the protective bush of the oil packing assembly for the centrifugal compressor, to the operational characteristics of which high requirements are imposed. These characteristics are the wear resistance, fatigue limit and ductility for accomplishing the press fitting on the shaft. 
However, the samples of steel $40 \mathrm{Cr}$ processed by the electro-spark carbonizing combined with the ultrasonic surface hardening showed higher wear resistance (by 1.2 times for steel $40 \mathrm{Cr}$ ) in comparison with the standard part. It leads to a considerable reduction in prices in manufacturing the protective bushes.

Paper [17] describes the effect of combined laser-ultrasonic hardening process with laser heat treatment. In this study a medium carbon and chromium tool steels were heat treated by a laser and then the specimen surfaces were severely deformed by an ultrasonic tool. This complex surface modification provides more than triple increase in micro hardness and formation the compressive residual stresses in the surface layer with simultaneous formation of the regular surface microrelief with minimum surface roughness and high oil holding capacity.

Thus, the application of the ultrasonic surface hardening gives the possibilities of a considerable reduction in the production costs of machine parts, which allows obtaining a higher economic effect or better performance characteristics.

\section{Conclusions}

Taking into consideration the analysis of the research conducted in the sphere of the ultrasonic surface hardening, one can make the following conclusions:

1. The ultrasonic surface hardening can be considered as a universal method of providing the improved operational characteristics of parts. The interest in the ultrasonic surface hardening is taken in obtaining nano- and submicrocrystal structures of the surface layer, activating the surface before the thermochemical processing and providing a favorable geometry before coating.

2. The application of the ultrasonic surface hardening to provide the improved properties of the typical construction materials is considered to be perspective. Such an approach allows using widely spread materials as a replacement for the materials of a special use, which simplifying the production.

3. The knowledge gained in the sphere of the ultrasonic surface hardening even now allow creating the mathematical models providing the calculation of the most rational processing modes depending on the desired result, which allows increasing the efficiency of using the ultrasonic surface hardening.

4. The ultrasonic processing is used in restoring the details. The possibility of using the multi-purpose equipment for conducting the ultrasonic surface hardening and the presence of valid models for calculating the rational processing modes can significantly increase the sphere of applying the ultrasonic deforming.

5. Material nano-constructing by the ultrasonic surface hardening contributes to maintaining the properties and the structure of the detail surface layer at high temperatures, which can increase the part durability in severe temperature modes. 


\section{References}

1. Kh. M. Rakhimyanov, Yu.S. Semyonova, Strengthening technologies and coatings 62, 3 (2010)

2. Kh. M. Rakhimyanov, Yu. S. Semenova, A. S. Eremina, IOP Conference Series: Materials Science and Engineering, 253, 012030 (2017)

3. Qi Fang Zhu, Ze Ming Sun Ma Tongda Vasily A Klimenov Viacheslav Borozna, Bao Hong Zhu. Materials Science Forum, 654:656, 1892 (2010)

4. Y. Li,D. Wang,B. Gong, C. Deng, Surface Engineering, 33, 12, 903 (2017)

5. Seky Chang, Young-Sik Pyun and Auezhan Amanov. Materials. 10:2-188·(2017)

6. V.A. Klimenov, Z.G.Kovalevskaya, V.Y. Ul'yanitskii, K.V. Zaitsev, V.Y. Borozna, Welding International. 24, 10, 803, (2010)

7. A.V. Panin, A.R. Shugurov, M.S. Kazachenok, V.P. Sergeev, Technical Physics. 57:6, 779, (2012)

8. A.V. Panin, E.A. Melnikova, O.B. Perevalova, Yu.I. Pochivalov, M.V. LeontyevaSmirnova, V.M. Chernov, Yu.F. Ivanov, Physical Mesomechanics, 12:3-4, 150 (2009)

9. A.V. Makarov, R.A. Savrai, E.S. Gorkunov, A.S. Yurovskikh, I.Y. Malygina, N.A. Davydova, Physical Mesomechanics, 18:1, 43 (2015)

10. L.B. Botaeva, V.A. Klimenov, V.I. Vereshagin, T.S. Petrovskaya, V.P. Ignatov, 8th Korea-Russia International Symposium on Science and Technology, KORUS 2004, 3, 99 (2005)

11. E.E. Kornienko, D.O. Mul', O.A. Rubtsova, S.P. Vaschenko, V.I. Kuzmin, I.P. Gulyaev, D.V. Sergachev, Thermophysics and Aeromechanics, 23:6, 919, (2016)

12. Zajcev K.V. Mining information and analytical bulletin., 6, 350 (2014)

13. M.Kh. Mukhametrakhimov. Rev. Adv. Mater. Sci., 31, 174 (2012)

14. V.A. Klimenov, Zh. G. Kovalevskaya, O. B. Perevalova, Yu. F. Ivanov, V. A. Kukareko, The Physics of Metals and Metallography, 102:6, 578 (2006)

15. M.S. Kazachenok, A.V. Panin, Yu.F. Ivanov, Yu.I. Pochivalov, R.Z. Valiev, Fizicheskaya Mezomekhanika. 8:4, 37 (2005)

16. S.I. Kryshtopa, D.Yu. Petryna, I. M.Bogatchuk, I.B. Prun'ko, V. M. Mel'nyk, Materials Science 53:3, 351 (2017)

17. D. Lesyk, S. Martinez, B. Mordyuk, V. Dzhemelinskyi, O.Danyleiko, Advances in Design, Simulation and Manufacturing. DSMIE 2018, 97 (2019) 